\title{
Treatment of Axillary Osmidrosis Using a Subcutaneous Pulsed Nd-YAG Laser
}

\author{
Daejin Kim, Junhyung Kim, Hyeonjung Yeo, Hyukjun Kwon, Daegu Son, Kihwan Han \\ Department of Plastic and Reconstructive Surgery, Keimyung University School of Medicine, Daegu, Korea
}

Background Axillary osmidrosis is characterized by an unpleasant odor, profuse sweating, and in some instances, staining of clothes that may socially and psychologically impair affected individuals. Various types of surgical procedures have been developed for the treatment of axillary osmidrosis. This study was undertaken to evaluate the effectiveness of subcutaneous pulsed neodymium: yttrium-aluminum-garnet (Nd-YAG) laser treatment for the treatment of axillary osmidrosis.

Methods Twenty-nine patients with axillary osmidrosis were included in this study. Patients were categorized according to the results of an axillary malodor grading system, and a subcutaneous pulsed Nd-YAG laser was applied to all patients. The treatment area for the appropriate distribution of laser energy was determined using the iodine starch test (Minor's test) against a grid pattern composed of $2 \times 2 \mathrm{~cm}$ squares. The endpoint of exposure was 300 to $500 \mathrm{~J}$ for each grid, depending on the preoperative evaluation results. The results were evaluated by measurement of axillary malodor both pre- and postoperatively using the grading system and iodine starch test.

Results The average follow-up period was 12.8 months. Nineteen patients had a fair-togood result and ten patients had poor results. The postoperative Minor's test demonstrated that there were remarkable improvements for patients with mild to moderate symptoms. Complications including superficial second degree burns $(n=3)$ were treated in a conservative manner. A deep second degree burn $(n=1)$ was treated by a surgical procedure.

Conclusions Subcutaneous pulsed Nd-YAG laser has many advantages and is an effective noninvasive treatment for mild to moderate axillary osmidrosis.

Keywords Lasers / Axilla / Starch
Correspondence: Junhyung Kim Department of Plastic and Reconstructive Surgery, Keimyung University School of Medicine, 56 Dalseong-ro, Jung-gu, Daegu 700-712, Korea

Tel: +82-53-250-7635

Fax: +82-53-255-0632

E-mail: med69@dsmc.or.kr
No potential conflict of interest relevant to this article was reported.

\section{INTRODUCTION}

Axillary osmidrosis does not affect physical health, but it is a hereditary condition where individuals may feel uncomfortable with odors emitted by the apocrine sweat glands of their axilla. The odor associated with axillary osmidrosis is caused by the oxidation and hydrolysis of $\varepsilon$-3-methyl 2-hexenoic acid carried out by the normal flora (staphylococcus aureus, gram negative bacilli) which exist on the apocrine sweat gland [1]. These bacterial metabolic processes are accelerated by exercise,exposure to heat, and emotional stress. Fatty acids, proteins, and carbohydrates are sources of $\varepsilon$-3-methyl 2-hexenoic acid. The potential

Copyright $(\odot 2012$ The Korean Society of Plastic and Reconstructive Surgeons

This is an Open Access article distributed under the terms of the Creative Commons Attribution Non-Commercial License (http://creativecommons.org/

licenses/by-nc/3.0/) which permits unrestricted non-commercial use, distribution, and reproduction in any medium, provided the original work is properly cited.

www.e-aps.org 
results of axillary osmidrosis include depression, anxiety and various negative social impacts [2].

This condition causes the individual slight discomfort in daily living and/or problems in their social life. Various procedures are under investigation. Treating osmidrosis with surgical resection of the apocrine glands is one effective and irreversible method. New treatment methods with at low relapse rate and that reduce the amount of scarring and temporary debilitation associated with surgery are in demand.

Proper diagnosis is required for identifying the appropriate treatment of axillary osmidrosis. Using the method introduced by Park and Shin [3], the iodine starch test was used in this study to determine the degree of severity [4], and laser irradiation was performed based on the results. Park and Shin [3] created classification tools to objectivize the degree of axillary osmidrosis. Based on the degree of severity, patients were treated with subcutaneous pulsed neodymium: yttrium-aluminumgarnet (Nd-YAG) laser [5], and we report the resulting efficacies and observed complications associated with specific grades of diagnosed axillary osmidrosis. Preoperative and postoperative differences were evaluated to identify relapse.

\section{METHODS}

\section{Patients}

From January 2010 to February 2011, of the 52 patients who visited a plastic surgery clinic reporting conditions of axillary osmidrosis, 29 total individuals (female, 15; male, 14) were selected to be treated with subcutaneous pulsed Nd-YAG laser. Thirty-three patients were excluded from the study because they did not want an Nd-YAG laser to be used $(n=28)$, or were lost to follow-up $(n=5)$. The ages of the individuals ranged from 11 to 45 years of age (mean, 23.4 years), and the follow-up period ranged from 6 to 18 months with a mean follow-up period of 12.8 months.

\section{Preoperative and postoperative evaluation}

Prior to operation, the severity of axillary osmidrosis was determined by the use of a questionnaire which examined perceived levels of malodor and sweating, and use of the classification system defined by Park and Shin [3] (Table 1, Figs. 1, 2). The questionnaire responses regarding malodor and sweating were based on subjective data from the patient as well as their family and friends. The grade was classified into one of three categories: minimal, moderate, and severe.

Table 1. Osmidrosis grading system

\begin{tabular}{|c|c|}
\hline Grade & Degree of malodor \\
\hline 0 & Gauze rubbed on the armpit does not give off any malodor under any circumstances (normal) \\
\hline 1 & $\begin{array}{l}\text { Gauze rubbed on the armpit emits slight malodor only when patients are under stressful conditions (exercising or walking) and no malodor } \\
\text { is detected from the rubbed gauze after performing usual daily activities }\end{array}$ \\
\hline 2 & $\begin{array}{l}\text { Gauze rubbed on the armpit emits strong malodor after performing daily activities but malodor could not be detected at a distance of } \\
1.5 \mathrm{~m} \text { from the patient }\end{array}$ \\
\hline 3 & Without the use of gauze, prominent malodor could be detected easily from the body at a distance of $1.5 \mathrm{~m}$ during daily activity \\
\hline
\end{tabular}

\section{Fig. 1. Preoperative iodine starch test (Minor's test)}

Minor's solution and starch were applied on both axillae. After 10 to 15 minutes, the color changed from white to blue-black. (A) Preoperative view of a 13-year-old female with grade 2 osmidrosis. (B) Preoperative view of a 17-year-old male patient with grade 3 osmidrosis showing more deeply discolored areas than the patient on the left.
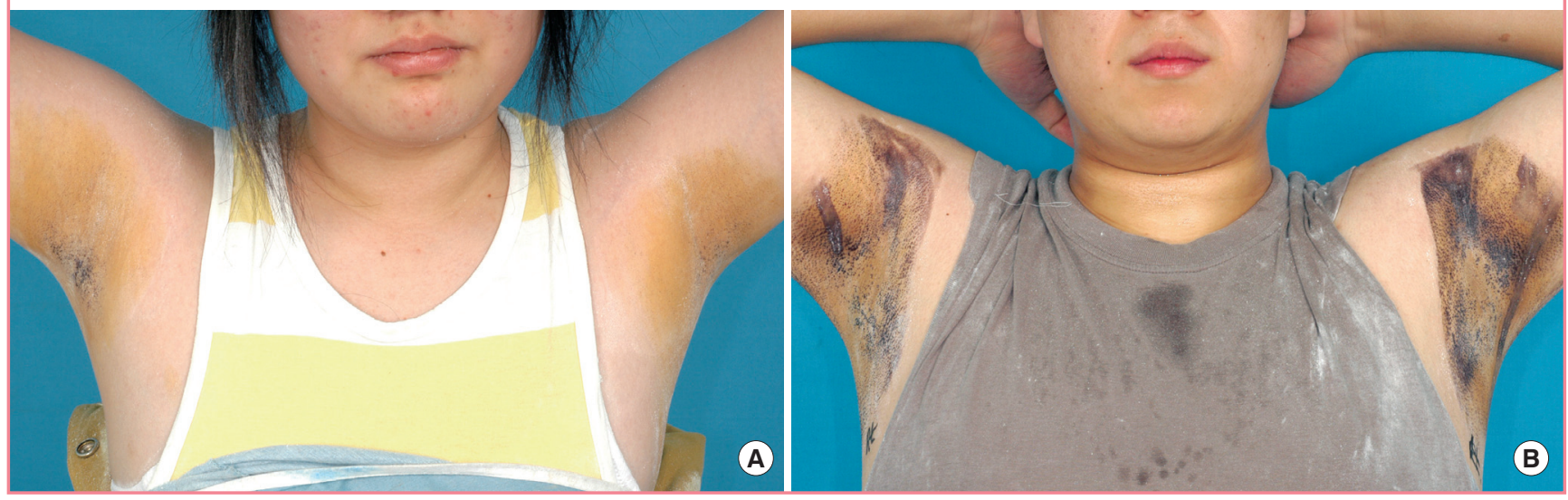
Mild osmidrosis was defined as an indistinct malodor and sweating which is unnoticed by others during normal daily activities, moderate osmidrosis was defined as malodor that could be identified by the patient but could not be detected by others at a distance of $1.5 \mathrm{~m}$ from the patient and accompanied by a little perspiration, and severe malodor and sweating could be detected easily from the body by others at a distance of $1.5 \mathrm{~m}$ during normal daily activity.

In order to reduce the uneven distribution of laser energy, a grid pattern composed of $2 \times 2 \mathrm{~cm}$ squares was drawn on the preoperative bilateral axillae. This was followed by the iodine starch test to identify the preoperative sweating pattern. The iodine starch test (Minor's test) [4] was used to objectively determine the surface area required for the operation. Areas of deep discoloration resulting from the iodine starch test were

\section{Fig. 2. Preoperative design}

Circle affected area with surgical marker, then mark a $2 \times 2 \mathrm{~cm}$ square grid pattern. Deeply discolored areas are marked with additional red lines.

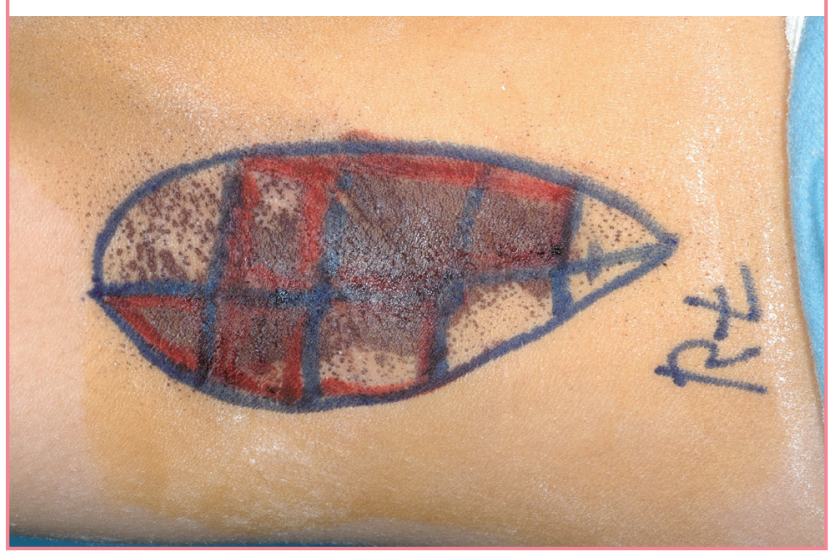

noted and the preoperative bilateral axillae were marked with a different color to make this area distinct (Fig. 2).

Satisfaction was evaluated after the operation using a questionnaire regarding any complications (scarring, burns, hematoma, loss of hair), degree of malodor, and sweating. The iodine starch test [4] was also used to perform a comparison of the preand postoperative area of sweating.

Statistical analysis was performed using SPSS ver. 16.0 (SPSS Inc., Chicago, IL, USA) on MS Windows, with statistical significance $(\mathrm{P}<0.05)$ determined using a paired t-test.

\section{Operative methods}

With the patients holding their arms in maximum elevation to expose both axillae, both axillae were draped and $0.005 \%$ chlorhexadine and $70 \%$ alcohol were applied. To relieve anxiety, 0.05-0.1 mg/kg of ketamine (Ketamine HCL, Huons, Seoul, Korea, $500 \mathrm{mg}, 10 \mathrm{~mL}$ ) and $2 \mathrm{mg} / \mathrm{kg}$ midazolam (Dormicum, Roche, Switzerland, $5 \mathrm{mg}, 5 \mathrm{~mL}$ ) was administered intravenously. Then a 1:100,000 mixture of epinephrine and 1\% lidocaine was mixed with normal saline in a 1:1 ratio. The subcutaneous injection was applied to the axilla followed by 5 minutes of compression. After making a hole using an 18-gauge needle, pretunneling was performed using a blunt dissector (Fig. 3A). Dissection was necessary so that the 1-mm diameter laser cannula could be inserted close to the subcutaneous apocrine gland targets. The cannula was inserted into the dermal-subdermal junction. The area of transcutaneous illumination indicated the positioning of the tips. The pulsed Nd-YAG laser (BL 3500N, B\&B Systems, Seoul, Korea) treatment was performed using a wavelength of $1,064 \mathrm{~nm}$, a pulse duration of $40 \mathrm{~Hz}$ and a power of $150 \mathrm{~mJ}$. The endpoints of exposure for each grid were 200 to

\section{Fig. 3. Operative technique}

(A) Multiple puncture points. Typically, three small punctures were used, but, random selection was possible. Adequate multiple punctures aided the method. Punctures were made with an 18-gauge needle at the anterior, posterior, proximal region, and border of each axilla. (B) Lipolysis was performed using an neodymium: yttrium-aluminum-garnet laser on the axilla. Laser exposures were 300 to $500 \mathrm{~J}$ for each square depending on the preoperative evaluation results.

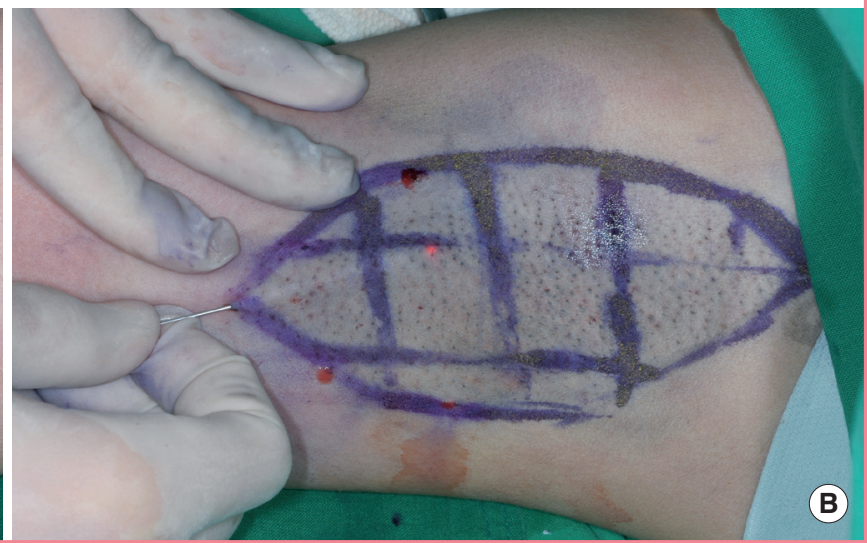


$300 \mathrm{~J}$ in grade 2 cases and $500 \mathrm{~J}$ in grade 3 cases, depending on the thickness of the skin, the density of the hair and the degree of severity of axillary osmidrosis (Fig. 3B). Laser assisted liposuction was not applied to objectively evaluate the effects of the treatment using only the Nd-YAG laser. No suture or drain was inserted into the cannula insertion site.

\section{Postoperative management}

Unlike other operative procedures, subcutaneous pulsed NdYAG laser does not require a specific postoperative dressing. Antibiotic ointment ( $0.3 \%$ ofloxacin ophthalmic ointment, Samil Pharm, Seoul, Korea, $3.5 \mathrm{~g}$ ) and hydrocellular foam (Allevyn standard, Smith \& Nephew, Largo, FL, USA) was applied to the wounds. This was an outpatient procedure, no restric-

\section{Table 2. Postoperative evaluation $(n=29)$}

\begin{tabular}{|c|c|}
\hline Postoperative evaluation & No. $(\%)$ \\
\hline $\begin{array}{l}\text { Subjective assessment } \\
\text { Good } \\
\text { Fair } \\
\text { Poor }\end{array}$ & $\begin{array}{r}13(44.8) \\
6(20.7) \\
10(34.5)\end{array}$ \\
\hline $\begin{array}{l}\text { Osmidrosis grading system } \\
\text { Grade 0, } 1 \text { (preoperatively/postoperatively) } \\
\text { Grade } 2 \\
\text { Grade } 3\end{array}$ & $\begin{array}{c}0(0) / 15(51.7) \\
19(65.5) / 4(13.8) \\
10(34.5) / 10(34.5)\end{array}$ \\
\hline $\begin{array}{l}\text { lodine starch test (Minor's test) } \\
\text { (mean squares of sweating area) } \\
\text { (total/grade 2/grade 3) } \\
\text { Preoperatively } \\
\text { Postoperatively }\end{array}$ & $\begin{array}{c}21.9 / 15.3 / 35.2 \\
12.5^{\mathrm{a}} / 5.3^{\mathrm{a})} / 26.2\end{array}$ \\
\hline $\begin{array}{l}\text { Complication } \\
\text { Burn or skin erosion } \\
\text { Scar } \\
\text { Hematoma or infection } \\
\text { Movement limitation } \\
\text { Major vessel or nerve injury }\end{array}$ & $\begin{array}{l}4(13.8) \\
0(0) \\
0(0) \\
0(0) \\
0(0)\end{array}$ \\
\hline
\end{tabular}

tions in arm movements were required, and patients were able to return immediately to everyday life. The dressing remained in place overnight and was removed the day after the operation. After removal of the dressing, patients performed a simple exercise and then showered.

\section{RESULTS}

There were no observations of postoperative hematoma, necrosis, infection, or axillary hair loss in any of the patients (Table $2)$. The most common complications were contact burns $(n=4$, $13.8 \%$ ) caused by the laser cannula. Of these contact burns, most were superficial second-degree burns. However, one case progressed from a superficial to a deep second degree burn, which subsequently necessitated a burn scar operation (Fig. 4).

Every patient involved in the operation was assessed to have grade 2 or 3 osmidrosis (grade $2, \mathrm{n}=19$; grade $3, \mathrm{n}=10$ ) (Table

\section{Fig. 5. Preoperative and postoperative evaluation}

Distribution of axillary osmidrosis by preoperative and postoperative evaluation, according to the osmidrosis grading system.

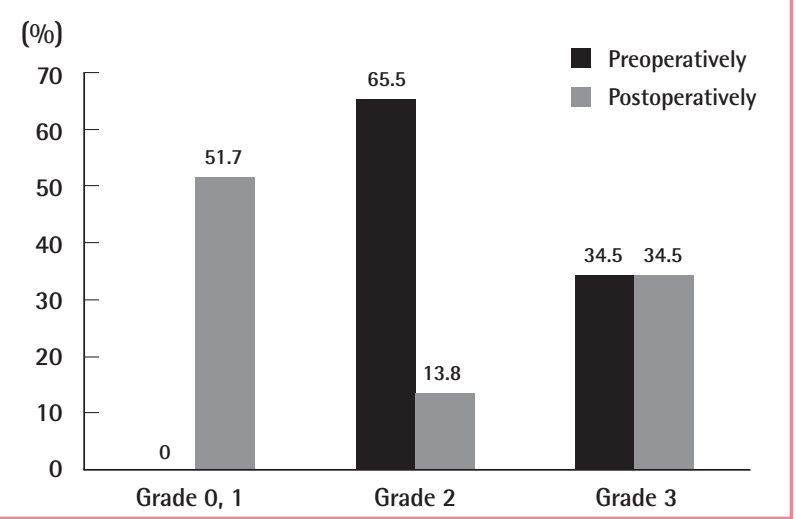

\section{Fig. 4. Postoperative complications viewed after 10 days}

(A) A 36-year-old female showed superficial second degree burns on the right axilla and was treated in a conservative manner. (B) A 40-year-old male showed deep second degree burns on the left axilla and was treated with excision and primary closure.
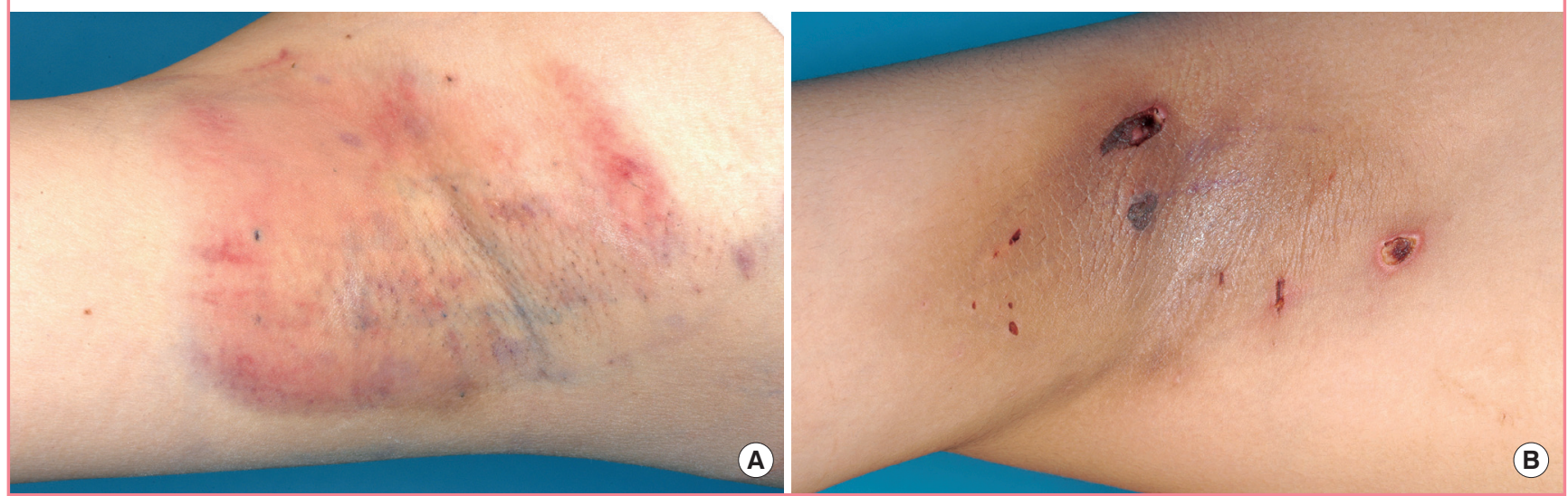


\section{Fig. 6. The postoperative iodine starch test (Minor's test)}

Minor's test showing a remarkable improvement. (A) Preoperative view of a 30-year-old male. (B) Eight months after subcutaneous pulsed neodymium: yttrium-aluminum-garnet laser treatment.
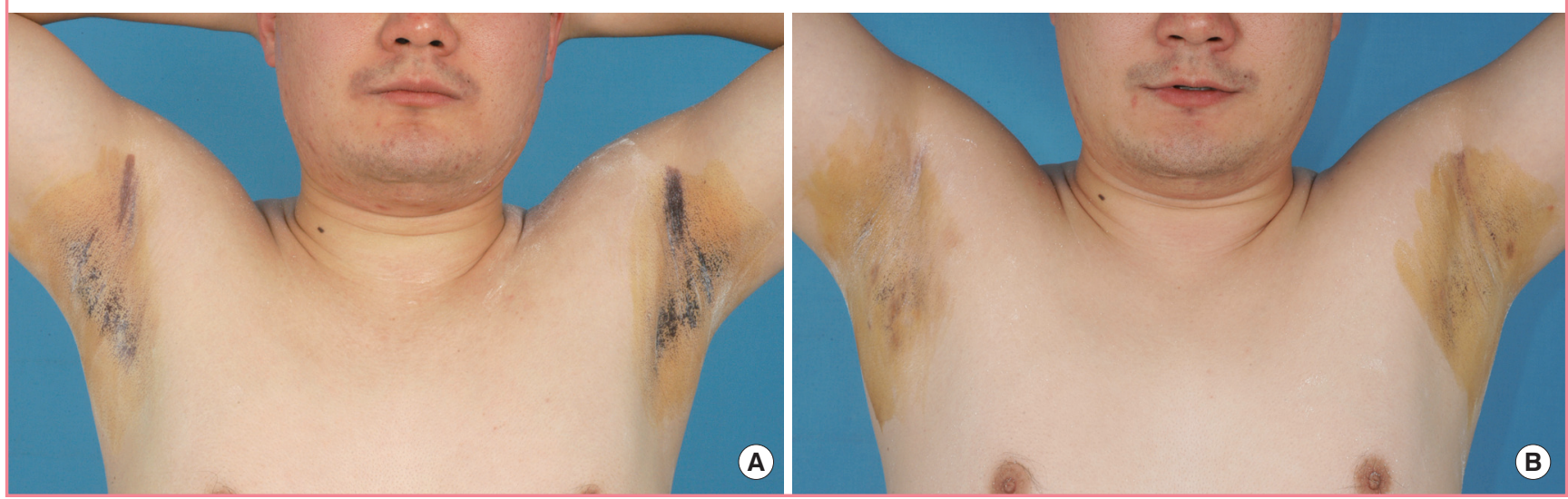

2, Fig. 5). Out of 19 grade 2 patients, 13 (44.8\%) felt good after the operation and recovered well without complications or relapse. Six patients (20.7\%) felt fair after the operation. These patients reported that some of the malodor remained but that it was not severe enough to treat by a second operation. The remaining ten patients (34.5\%), comprising one woman and nine men, showed poor results. All of these patients were grade 3 cases (Table 2, Fig. 5) and reported relapse of osmidrosis 6 months after the operation. Follow-up treatment for these patients included dermal shavings for three (30\%) patients and ultrasound assisted liposuction (UAL) for four (40\%) of the patients.

The iodine starch test [4] was used to evaluate the postoperative sweating surface of the axilla of each patient, again using a grid pattern composed of $2 \times 2 \mathrm{~cm}$ squares. In grade 2 patients, the number of squares reduced by the treatment was from 15.3 to 5.3 squares, which was statistically significant (Table 2 ). These patients also demonstrated an subjective reduction in discoloration (Fig. 6). For patients in grade 3, the number of squares was reduced from 34.2 to 26.2. However, this result was not statistically significant (Table 2).

\section{DISCUSSION}

The iodine starch test [4] as introduced by Bisbal et al. [6] to evaluate areas of sweating, is a useful method for evaluating the symptoms of both osmidrosis and bromhidrosis, but these conditions are different and their relationship to each other is not clearly defined. Osmidrosis, which is directly related to sweating, is in many cases accompanied by bromhidrosis [7]. The degree of severity of axillary osmidrosis is proportional to the amount of sweating, and the iodine starch test [4] has been suc- cessfully used to accurately measure results in previous studies [8]. Previous authors have used preoperative and postoperative physical examinations to evaluate the effects of the treatment, and subjectively evaluated the severity of osmidrosis and degree of sweating by visual means. Their study data helped us to determine the power and range of the irradiation (total laser energy) to use in our clinical procedure. Additionally, compliance was easier to achieve because the patient observed the change after the operation. In our study, the total laser energy utilized varied from 4,500 to $17,500 \mathrm{~J}$. Therefore, the total laser energy of each grid is important for reducing the heterogeneous distribution of laser energy. The exposure ranges for each grid were 200 to $300 \mathrm{~J}$ in grade 2 cases and 200 to $500 \mathrm{~J}$ in grade 3 cases, depending on the thickness of the skin, the density of the hair and the degree of severity of axillary osmidrosis, which were used to modify the Ichikawa et al. [5] method.

Treatments for axillary osmidrosis are classified as either invasive or noninvasive. Current noninvasive methods involve the use of a 532-nm frequency-doubled Q-switched Nd-YAG laser, as introduced by Kunachak et al. [9]. This method is controversial due to the poor efficacy concerns related to a high reported rate of recurrence of osmidrosis. Invasive methods include dermal shaving [10], liposuction [2], $\mathrm{CO}_{2}$ laser [11], and UAL [12], but due to the significant risk for postoperative pain, flap necrosis, formation of hematoma, scars, and the possibility of postoperative mobility disorder from scar contracture or other postoperative discomfort, these invasive methods are typically reserved for refractory cases [13].

Ichikawa et al. [5] demonstrated that adipocytes can be destroyed by direct laser irradiation as evidenced by electron microscope histological data. This advancement has demonstrated that a laser could be used in the treatment of axillary osmidrosis. 
However, the thin cannula of $\mathrm{CO}_{2}$ lasers make them inefficient as a primary treatment, and they are instead only used to burn remnant apocrine glands after the primary procedure. Moreover, a direct axillary incision is required for use of $\mathrm{CO}_{2}$ laser irradiation, and there are commonly side effects such as flap elevation, formation of hematoma and seroma, wound dehiscence, and flap necrosis [11].

The 532-nm frequency-doubled Q-switched Nd-YAG laser has a lower power than other lasers used for treatment, and subsequently, proof of efficacy has been of particular interest.

Our study used a high power $(1,064 \mathrm{~nm}, 40 \mathrm{~Hz}, 150 \mathrm{~mJ})$ pulsed Nd-YAG laser that provided relatively stable radiation and low complication rates [14]. Kunachak et al. [9] reported that Nd-YAG laser radiation can penetrate 4-6 nm deeper into the subcutaneous layer than the $\mathrm{CO}_{2}$ laser. The intensity of radiation reaching surrounding tissues is less and unnecessary injury is thus reduced. The light energy emitted by the Nd-YAG laser is selectively absorbed by melanin and hemoglobin. The apocrine gland itself does not contain melanin but the adjacent hair shaft and root contains large amounts of melanin, thus attracting the energy of the laser irradiation and thereby destroying the apocrine gland blood supply [9].

Apocrine glands are more widely distributed in the subcutaneous layers than in the dermal layers, and appear predominantly in the border of the subdermal layer. Existing noninvasive methods mainly attempt to destroy the apocrine glands in the subcutaneous layer, but are not effective in preventing relapse. Patients of axillary osmidrosis typically have many granular tissues with thick layers. Our study removed subdermal apocrine glands and reduced the number of granules through direct irradiation using a pulsed Nd:YAG laser tunneling through incisions in subcutaneous tissues. Most importantly, the accurate locations of the cannula tips were identified by the intensity of the transcutaneous illumination, so further research is needed to determine the appropriate depth of the cannulae. Byeon et al. [15] reported that the middle portion of the axilla has the largest and highest density of apocrine glands. They also reported that the apocrine glands in the middle of the axilla area reside deep in the subcutaneous layers. The higher relative density of apocrine glands makes the center of the axilla the most obvious target for treatment. However, the central region of the axilla also has an uneven surface, and this makes tunneling through the tissues more difficult. Patients were instructed to perform full extension of the arms to prevent injuries to the major structures of the axilla during the laser treatment. Our study dissected deeper into the central area to access the deeper glands located there.

In the study, the ten relapsed cases were all preoperatively identified as grade 3. A study by Ichikawa et al. [5] reported that every patient was satisfied with their method, which was very similar to the method conducted by this author. However, in the experiment of Ichikawa et al. [5], the severity of axillary osmidrosis was not classified and there were only three men out of a total of seventeen patients. It is our observation that axillary osmidrosis in men is usually more severe and more difficult to treat than in women.

The reason our study had higher relapse rates seems to be that most of the male participants $(n=14)$ involved in the experiment had relatively large areas requiring operative treatment $(\mathrm{n}=12)$ (Fig. 1). Also, out of ten grade 3 patients, nine were men. The axilla was divided into square shapes by preoperative evaluation (Fig. 2). Grade 3 patients had an average of 34.2 affected squares of the treatment surface as determined by the iodine starch test (Minor's test) [4], whereas the other patients had an average of 18.9 squares. The grade 3 group's treatment area exceeded the grade 2 group's by 15.3 squares, and the discoloration was worse in grade 3 than grade 2 (Fig. 1). In the authors' experience, the men were difficult to irradiate evenly because they had thick skin, which was difficult to dissect.

All grade 3 patients exhibited relapse of axillary osmidrosis and were unsatisfied with the treatment results. Subcutaneous pulsed Nd-YAG laser could be an effective treatment for grade 1 and 2 axillary osmidrosis cases as the methods are easy and postoperative care is minimal, allowing patients to quickly return to the activities of daily living. Ichikawa et al. [5] reported that the operator's skill is important in applying irradiation under the skin. They also reported that flap necrosis, hematoma formation, scarring, and nerve damage are also possible. Our study experienced four (13.8\%) cases of simple burn injuries. Such burn injuries occur when the laser cannula tips are located too close to the skin surface, so ensuring appropriate cannula location is very important during the irradiation dosing. Cooling the surface of the treated area during the procedure using normal saline could be helpful to prevent burn injuries.

Out of twenty-nine patients, ten (34.5\%) had relapsed 6 months after the operation, and all of these patients were grade 3 cases. These cases were revised with UAL or dermal shaving, instead of with an Nd-YAG laser. Patients with axillary osmidrosis at or below grade 2 had no relapse. However, if these cases relapsed, revision with Nd-YAG laser might be feasible.

In conclusion, use of the Nd-YAG laser for treatment of axillary osmidrosis has many advantages and its efficacy for treatment of grade 1 and 2 axillary osmidrosis has been demonstrated. With surgeons that are trained in the appropriate skills required, it is a recommended method of treatment. 


\section{REFERENCES}

1. Akutsu T, Sekiguchi K, Ohmori T, et al. Individual comparisons of the levels of (E)-3-methyl-2-hexenoic acid, an axillary odor-related compound, in Japanese. Chem Senses 2006;31:557-63.

2. Perng $\mathrm{CK}$, Yeh FL, Ma H, et al. Is the treatment of axillary osmidrosis with liposuction better than open surgery? Plast Reconstr Surg 2004;114:93-7.

3. Park YJ, Shin MS. What is the best method for treating osmidrosis? Ann Plast Surg 2001;47:303-9.

4. Swinehart JM. Treatment of axillary hyperhidrosis: combination of the starch-iodine test with the tumescent liposuction technique. Dermatol Surg 2000;26:392-6.

5. Ichikawa K, Miyasaka M, Aikawa Y. Subcutaneous laser treatment of axillary osmidrosis: a new technique. Plast Reconstr Surg 2006;118:170-4.

6. Bisbal J, del Cacho C, Casalots J. Surgical treatment of axillary hyperhidrosis. Ann Plast Surg 1987;18:429-36.

7. Mao GY, Yang SL, Zheng JH. Etiology and management of axillary bromidrosis: a brief review. Int J Dermatol 2008; 47:1063-8.

8. Davis PK. Surgical treatment of axillary hyperhidrosis. $\mathrm{Br} J$
Plast Surg 1971;24:99-100.

9. Kunachak S, Wongwaisayawan S, Leelaudomlipi P. Noninvasive treatment of bromidrosis by frequency-doubled Qswitched Nd:YAG laser. Aesthetic Plast Surg 2000;24:198201.

10. Qian JG, Wang XJ. Effectiveness and complications of subdermal excision of apocrine glands in 206 cases with axillary osmidrosis. J Plast Reconstr Aesthet Surg 2010;63:1003-7.

11. Kim IH, Seo SL, Oh CH. Minimally invasive surgery for axillary osmidrosis: combined operation with $\mathrm{CO}_{2}$ laser and subcutaneous tissue remover. Dermatol Surg 1999;25:875-9.

12. Hong JP, Shin HW, Yoo SC, et al. Ultrasound-assisted lipoplasty treatment for axillary bromidrosis: clinical experience of 375 cases. Plast Reconstr Surg 2004;113:1264-9.

13. Atkins JL, Butler PE. Hyperhidrosis: a review of current management. Plast Reconstr Surg 2002;110:222-8.

14. Ichikawa K, Tanino R, Wakaki M. Histologic and photonic evaluation of a pulsed Nd:YAG laser for ablation of subcutaneous adipose tissue. Tokai J Exp Clin Med 2006;31:136-40.

15. Byeon JH, Wee SS, Lim P. Histological location, size and distribution of apocrine glands in axillary osmidrosis. J Korean Soc Plast Reconstr Surg 1988;15:419-25. 